\title{
Changes in Glutamate/NMDA Receptor Subunit 1 Expression in Rat Brain after Acute and Subacute Exposure to Methamphetamine
}

\author{
Walailuk Kerdsan, Samur Thanoi, and Sutisa Nudmamud-Thanoi \\ Department of Anatomy, Faculty of Medical Science, Naresuan University, Phitsanulok 65000, Thailand \\ Correspondence should be addressed to Sutisa Nudmamud-Thanoi, sutisat@nu.ac.th \\ Received 26 February 2009; Accepted 23 April 2009 \\ Recommended by Laurence D. Fechter
}

Methamphetamine (METH) is a psychostimulant drug of abuse that produces long-term behavioral changes including behavioral sensitization, tolerance, and dependence. METH has been reported to induce neurotoxic effects in several areas of the brain via the dopaminergic system. Changes of dopamine function can induce malfunction of the glutamatergic system. Therefore, the aim of the present study was to examine the effects of METH administration on the expression of glutamate N-methylD-aspartate receptor subunit 1 (NMDAR1) in frontal cortex, striatum, and hippocampal formation after acute and subacute exposure to METH by western blotting. Male Sprague-Dawley rats were injected intraperitoneally with a single dose of $8 \mathrm{mg} / \mathrm{kg}$ METH, $4 \mathrm{mg} / \mathrm{kg} /$ day METH for 14 days and saline in acute, subacute, and control groups, respectively. A significant increase in NMDAR1 immunoreactive protein was found in frontal cortex in the subacute group $(P=.036)$ but not in the acute group $(P=.580)$. Moreover, a significant increase in NMDAR1 was also observed in striatum in both acute $(P=.025)$ and subacute groups $(P=.023)$. However, no significant differences in NMDAR1 in hippocampal formation were observed in either acute or subacute group. The results suggest that an upregulation of NMDA receptor expression may be a consequence of glutamatergic dysfunction induced by METH.

Copyright (C) 2009 Walailuk Kerdsan et al. This is an open access article distributed under the Creative Commons Attribution License, which permits unrestricted use, distribution, and reproduction in any medium, provided the original work is properly cited.

\section{Introduction}

Methamphetamine (METH), an N-methyl homolog of amphetamine, is an addictive psychostimulant with potent effects on the central nervous system, resulting in behavioral changes including sensitization, tolerance, and dependence [1]. Neurochemical studies have shown long-term neurotoxic effects of METH on monoamine neurotransmitters including dopamine [2], serotonin, and norepinephrine [3] systems in several brain regions. Moreover, many studies have suggested that not only are monoamine systems affected by METH-induced neurotoxicity, but the glutamatergic system has also been involved in the toxic effect of METH $[4,5]$.

Several studies support the role of glutamate in the development of METH toxicity. METH enhances glutamate release in several brain regions, such as striatum [6], cerebral cortex [7], hippocampus [8], ventral tegmental area, and nucleus accumbens [9]. An elevation of extracellular glutamate can activate glutamate receptors which may mediate excitotoxicity leading to apoptosis and/or neuronal cell death [10]. With Glutamate/N-methyl-Daspartate (NMDA) receptors, which play a principle role in excitatory neurotransmission, brain development, and synaptic plasticity, have been reported to be implicated in METH neurotoxicity [11]. Furthermore, a reduction of glutamate/NMDA receptors has been reported in substantia nigra, nucleus accumbens, and medial prefrontal cortex during amphetamine withdrawal [12]. These observations suggest that glutamate/NMDA receptors may play a critical role in the effects of drug addiction. However, the neurochemical and behavioral consequences of METH depend on the extent and route of exposure to the drug [13]. Changes in NMDA receptors seem to be related to the 
administration model and doses of METH $[12,14]$. It is, therefore, important to study effects on the glutamatergic system, particularly NMDA receptors, underlying the pattern of exposure to METH. Therefore, the aim of the present study was to investigate the effects of acute and subacute METH administration in an exposure pattern of single daily injection on the alteration of NMDA receptor subunit 1 immunoreactivity in rat striatum, frontal cortex and hippocampal formation.

\section{Materials and Methods}

Male Sprague-Dawley rats (250-280 g) were obtained from the National Animal Center, Mahidol University, Thailand. The animals were housed 2-3 per cage and maintained at $24 \pm 1{ }^{\circ} \mathrm{C}$ under a 12-hour light/dark cycle with free access to water and food. All animal procedures were carried out in compliance with Mahidol University Code of Practice and the National Institutes of Health (USA) Guidelines for treatment of laboratory animals. The protocol for this study was approved by the Animal Research Committee of Naresuan University, Thailand.

Drug treatment consisted of D-methamphetamine $\mathrm{HCl}$ (Alltech, Ill, USA) with permission of Ministry of Public Health. Animals in the acute METH group received vehicle for 13 days and followed by one injection of METH $(8 \mathrm{mg} / \mathrm{kg}$, IP) on day 14. Animals were treated with METH for 14 days $(4 \mathrm{mg} / \mathrm{kg} / \mathrm{day}, \mathrm{IP})$ in the subacute group. For the control group, animals were treated with vehicle for 14 days. All rats were decapitated at 24-hour after the last dose. The striatum, frontal cortex and hippocampal formation were isolated, and tissues were stored at $-70^{\circ} \mathrm{C}$ until used.

NMDA receptor subunit 1 immunoreactivity (NMDAR1-IR) was determined following the method previously reported [15]. Briefly, tissue was homogenized in $5 \mathrm{mM}$ Tris- $\mathrm{HCl}$ containing $20 \mathrm{mM} \mathrm{NaCl}, \mathrm{pH} \mathrm{8.0,} \mathrm{and}$ the homogenate was then centrifuged. The pellet was homogenized again in lysis buffer. Protein concentration in the tissue lysate was estimated by the bicinchoninic acid assay (BCA) (Pierce, Ill, USA). Tissue lysate was then boiled for 5 minutes with an equal volume of $2 \mathrm{x}$ sample buffer. The supernatants containing $50 \mu \mathrm{g}$ original proteins, chosen to give results within the linear range for NMDAR1 estimations, were separated by polyacrylamide gel electrophoresis. The proteins were then electrotransferred onto polyvinylidene fluoride (PVDF) membranes (Amersham, Ill, USA). Membranes were incubated with protein blocking solution and subsequently incubated with NMDAR1 antibody (Sigma-Aldrich, Mo, USA). The membranes were then incubated with biotinylated secondary antibody (Vector, Calif, USA) and avidin biotinylated horseradish peroxidase complexes (ABC kit) (Vector, Calif, USA). The protein immunoreactivity was visualized using 3,3',5,5' -tetramethylbenzidine (TMB) (Promega, USA). Immunoblotting for $\beta$-actin (Santa Cruz, Calif, USA) was used as an internal standard to confirm equal protein loading and sample transferring. Expression of NMDAR1 protein was normalized against that of $\beta$-actin.

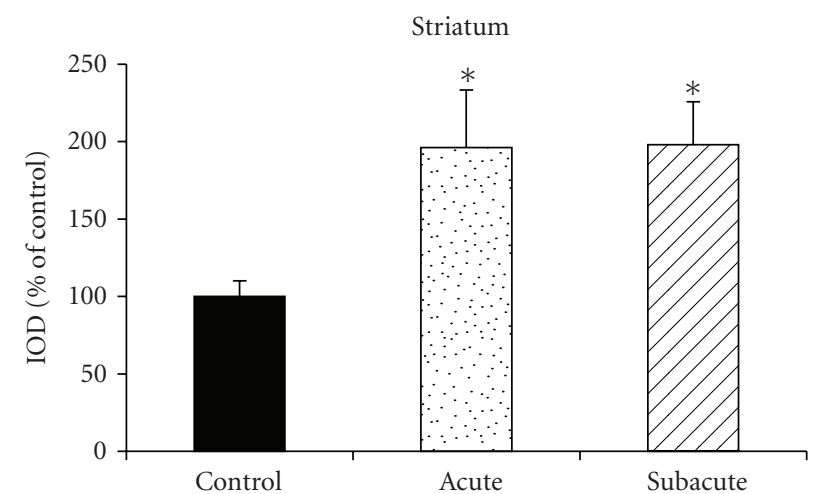

FIGURE 1: Expression of NMDAR1 in striatum. Data are integrated optical density of NMDAR1 immunoreactivity band, normalized to $\beta$-actin levels, and expressed as percentage of the control group. Values represent mean \pm S.E.M $(n=5-7) .{ }^{*} P<.05$ in comparison with the control group by one-way ANOVA with Dunnett post-hoc test.

The immunostained membranes were scanned into a computer and integrated optical density (IOD) was measured by Scion Image program based on NIH image (v. Alpha 4.0.3.2; http://www.scioncorp.com; 2000-2001). The value is the sum of the optical densities of all pixels in the region. All immunoreactive bands were measured with the same dimensions to obtain their integrated optical density. Data were expressed as percentage of control group and all data were represented as mean \pm S.E.M. Differences between groups were analyzed using ANOVA followed by Dunnett post-hoc test.

\section{Results}

Immunostaining for NMDAR1 consistently demonstrated an immunoreactive band corresponding to approximately $116 \mathrm{kDa}$ and a $\beta$-actin immunoreactive band was approximately $43 \mathrm{kDa}$. Densitometric analysis was performed to quantify the alteration of NMDAR1 expression relative to $\beta$-actin immunoreactivity. ANOVA demonstrated that NMDAR1-IR was significantly increased above control in the striatum after both acute $(P=.025)$ and subacute $(P=.023)$ METH administration, and reached $196 \%$ and $198 \%$ of control group, respectively (Figure 1). Moreover, a significant increase of NMDAR1-IR (508\% over control) was also observed in the frontal cortex in the subacute METH $(P=.036)$, but not in the acute group $(P=.580)$ (Figure 2$)$. However, there were no significant differences of NMDAR1IR in the hippocampal formation either in acute $(P=.839)$ or subacute $(P=.711)$ METH groups when compared with the control group (Figure 3 ).

\section{Discussion}

The present study revealed that single daily exposure to METH can induce a significant increase of NMDAR1-IR in the cortical and subcortical regions of rat brain. The effect was observed in the frontal cortex after subacute 


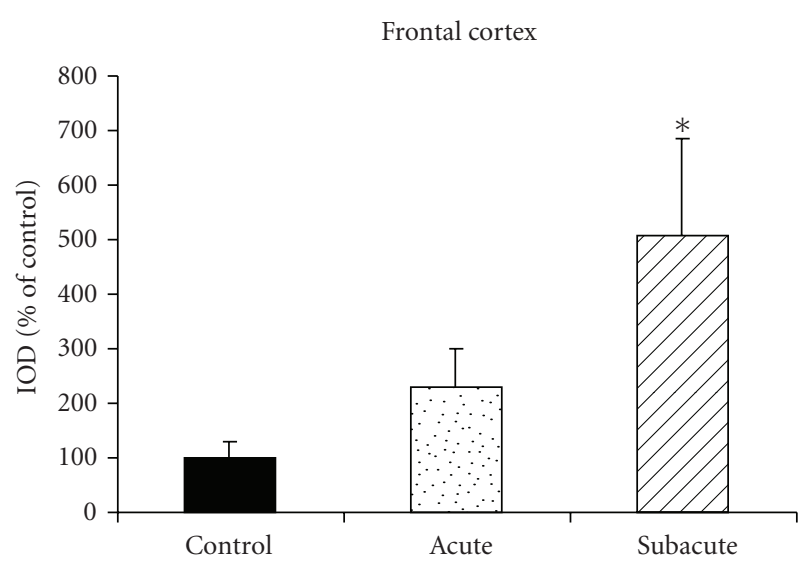

FIgURE 2: Expression of NMDAR1 in frontal cortex. Data are integrated optical density of NMDAR1 immunoreactivity band, normalized to $\beta$-actin levels and expressed as percentage of the control group. Values represent mean \pm S.E.M $(n=5-7) .{ }^{*} P<.05$ in comparison with the control group by one-way ANOVA with Dunnett post-hoc test.

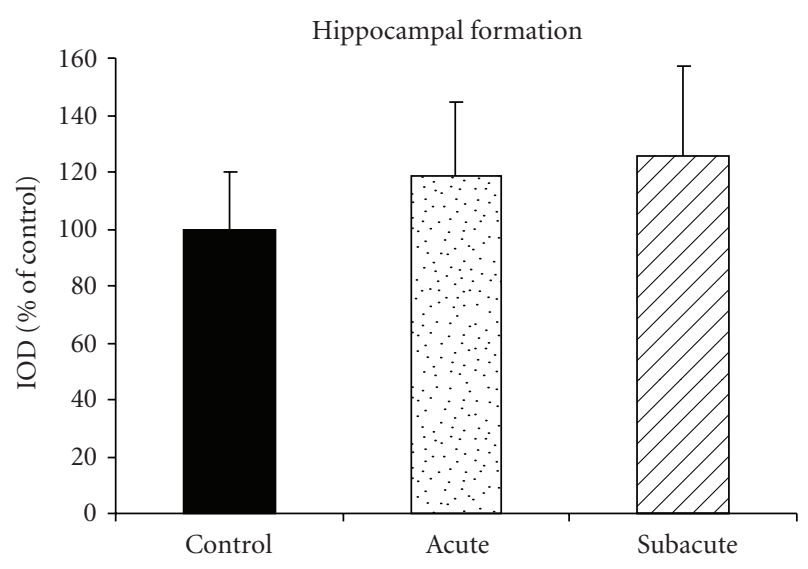

FIgURE 3: Expression of NMDAR1 in hippocampal formation. Data are integrated optical density of NMDAR1 immunoreactivity band, normalized to $\beta$-actin levels and expressed as percentage of the control group. Values represent mean \pm S.E.M $(n=5-7)$.

METH administration. Moreover, the results showed an upregulation of NMDAR1 in the striatum after both acute and subacute METH administrations. As METH is an abused psychostimulant, our results provide a support for effects of these drugs on glutamatergic systems. Glutamatergic dysfunctions may be a consequence of changes in the dopaminergic system induced by psychostimulants [16]. Moreover, an impairment of monoamine neurotransmission after repeated METH exposure can produce changes in amino acid homeostasis, potentially leading to neurotoxicity [17].

The results of this study are in agreement with previous reports of elevated NMDA receptor binding in cerebral cortex following both psychostimulants METH [18] and cocaine [19], suggesting the involvement of the NMDA receptor in the development of drug addiction.
Supporting a hypothesis of glutamatergic dysfunction in cortical and subcortical regions following psychostimulant drug exposure, we previously observed an upregulation of NMDAR1 density in dentate gyrus following administration of pseudoephedrine, a drug which is considered to be a psychostimulant [20]. Moreover, increased metabotropic glutamate receptor (mGluR5) protein and gene expression have been reported in cultured cortical and hippocampal neurons after amphetamine administration [21]. An increase in vesicular glutamate transporter (VGLUT1), an important component of glutamatergic neurons that regulates glutamate release, has been observed in striatal and cortical regions after METH administration, supporting evidence for METH-induced elevation of glutamate release in striatum and cortex [6].

Although no alteration in NMDAR1-IR in the hippocampal formation was observed in the present study after METH treatment, the finding is consistent with a previous study that revealed a slight decrease in NMDAR1 mRNA expression in dentate gyrus after exposure to cocaine [22]. Moreover, no differences in the NMDAR1, $2 \mathrm{~A}$ and $2 \mathrm{~B}$ immunoreactivities were observed in the hippocampus of METH-sensitized rats [23]. Taken together, our observations provide further support for the regional specificity of glutamatergic dysfunction after METH administration.

In summary, our results indicate an upregulation of NMDA receptor expression after acute and subacute exposure to METH reflecting the dysfunction of glutamatergic system. The alterations in the NMDAR1 found in the striatum and frontal cortex suggest that other components of the glutamate synapse may be abnormal in these regions in METH dependence. Further work is still needed to study other components of glutamatergic neurotransmission in order to investigate abnormalities of glutamatergic system in METH dependence.

\section{Acknowledgments}

This study was financially supported by Naresuan University Research Fund and Co-funding between the Thailand Research Fund and Commission on Higher-Education (TRF-CHE). W. Kerdsan was supported by a scholarship under the staff development project of Naresuan University, Payao Campus.

\section{References}

[1] A. Reid and A. Lingford-Hughes, "Neuropharmacology of addiction," Psychiatry, vol. 5, no. 12, pp. 449-454, 2006.

[2] S. J. O'Dell, F. B. Weihmuller, and J. F. Marshall, "Multiple methamphetamine injections induce marked increases in extracellular striatal dopamine which correlate with subsequent neurotoxicity," Brain Research, vol. 564, no. 2, pp. 256260, 1991.

[3] D. S. Segal and R. Kuczenski, "An escalating dose "binge" model of amphetamine psychosis: behavioral and neurochemical characteristics," Journal of Neuroscience, vol. 17, no. 7, pp. 2551-2566, 1997. 
[4] J. F. Marshall, S. J. O’Del, and F. B. Weihmuller, "Dopamineglutamate interactions in methamphetamine-induced neurotoxicity," Journal of Neural Transmission. General Section, vol. 91, no. 2-3, pp. 241-154, 1993.

[5] S. E. Stephans and B. K. Yamamoto, "Methamphetamineinduced neurotoxicity: roles for glutamate and dopamine efflux," Synapse, vol. 17, no. 3, pp. 203-209, 1994.

[6] K. A. Mark, M. S. Quinton, S. J. Russek, and B. K. Yamamoto, "Dynamic changes in vesicular glutamate transporter 1 function and expression related to methamphetamine-induced glutamate release," The Journal of Neuroscience, vol. 27, no. 25, pp. 6823-6831, 2007.

[7] J. R. Shoblock, E. B. Sullivan, I. M. Maisonneuve, and S. D. Glick, "Neurochemical and behavioral differences between $d$ methamphetamine and d-amphetamine in rats," Psychopharmacology, vol. 165, no. 4, pp. 359-369, 2003.

[8] J. Raudensky and B. K. Yamamoto, "Effects of chronic unpredictable stress and methamphetamine on hippocampal glutamate function," Brain Research, vol. 1135, no. 1, pp. 129135, 2007.

[9] C.-J. Xue, J. P. Ng, Y. Li, and M. E. Wolf, "Acute and repeated systemic amphetamine administration: effects on extracellular glutamate, aspartate, and serine levels in rat ventral tegmental area and nucleus accumbens," Journal of Neurochemistry, vol. 67, no. 1, pp. 352-363, 1996.

[10] J. L. Cadet, S. Jayanthi, and X. Deng, "Speed kills: cellular and molecular bases of methamphetamine-induced nerve terminal degeneration and neuronal apoptosis," The FASEB Journal, vol. 17, no. 13, pp. 1775-1788, 2003.

[11] T. Abekawa, T. Ohmori, and T. Koyama, "Effects of nitric oxide synthesis inhibition on methamphetamine-induced dopaminergic and serotonergic neurotoxicity in the rat brain," Journal of Neural Transmission, vol. 103, no. 6, pp. 671-680, 1996.

[12] W. Lu, L. M. Monteggia, and M. E. Wolf, "Withdrawal from repeated amphetamine administration reduces NMDAR1 expression in the rat substantia nigra, nucleus accumbens and medial prefrontal cortex," European Journal of Neuroscience, vol. 11, no. 9, pp. 3167-3177, 1999.

[13] C. Davidson, T. H. Lee, and E. H. Ellinwood, "Acute and chronic continuous methamphetamine have different longterm behavioral and neurochemical consequences," Neurochemistry International, vol. 46, no. 3, pp. 189-203, 2005.

[14] P. F. Simões, A. P. Silva, F. C. Pereira, et al., "Methamphetamine induces alterations on hippocampal NMDA and AMPA receptor subunit levels and impairs spatial working memory," Neuroscience, vol. 150, no. 2, pp. 433-441, 2007.

[15] S. Nudmamud-Thanoi and G. P. Reynolds, "The NR1 subunit of the glutamate/NMDA receptor in the superior temporal cortex in schizophrenia and affective disorders," Neuroscience Letters, vol. 372, no. 1-2, pp. 173-177, 2004.

[16] R. Karler, D. K. Thai, and L. D. Calder, "Interactions of dopamine, glutamate and GABA systems in mediating amphetamaine-and cocaine-induced stereotypy and behavioral sensitization," in Glutamate and Addiction, B. H. Herman, J. Frankenheim, R. Litten, P. H. Sheridan, F. F. Weight, and S. R. Zukin, Eds., pp. 107-125, Humana Press, New Jersey, NJ, USA, 2003.

[17] D. Bustamante, Z.-B. You, M.-N. Castel, et al., "Effect of single and repeated methamphetamine treatment on neurotransmitter release in substantia nigra and neostriatum of the rat," Journal of Neurochemistry, vol. 83, no. 3, pp. 645-654, 2002.
[18] A. J. Eisch, W. Marshall, W. Slikker, et al., "Methamphetamineinduced damage to cortical neurons is indicated by a newlydeveloped fluorescent tag," Society for Neuroscience Abstracts, vol. 22, p. 1915, 1996.

[19] Y. Itzhak, "Modulation of the PCP/NMDA receptor complex and sigma binding sites by psychostimulants," Neurotoxicology and Teratology, vol. 16, no. 4, pp. 363-368, 1994.

[20] S. Nudmamud-Thanoi, S. Thanoi, and P. Sobhon, "Increase of glutamate/ $N$-methyl-D-aspartate receptor immunodensity in the dentate gyrus of rats following pseudoephedrine administration," NeuroToxicology, vol. 27, no. 4, pp. 623-627, 2006.

[21] M.-F. Yu, T.-Y. Lin, W.-H. Ho, and H.-S. Yin, "Amphetamine induces differential changes in the gene expression of metabotropic glutamate receptor 5 in cultured cortical and hippocampal neurons," Journal of Molecular Neuroscience, vol. 17, no. 1, pp. 13-24, 2001.

[22] J. Turchan, M. Maj, and B. Przewlocka, "The effect of drugs of abuse on NMDAR1 receptor expression in the rat limbic system," Drug and Alcohol Dependence, vol. 72, no. 2, pp. 193196, 2003.

[23] H. Yamamoto, N. Kitamura, X.-H. Lin, et al., "Differential changes in glutamatergic transmission via $\mathrm{N}$-methyl$\mathrm{D}$-aspartate receptors in the hippocampus and striatum of rats behaviourally sensitized to methamphetamine," The International Journal of Neuropsychopharmacology, vol. 2, pp. 155-163, 1999. 

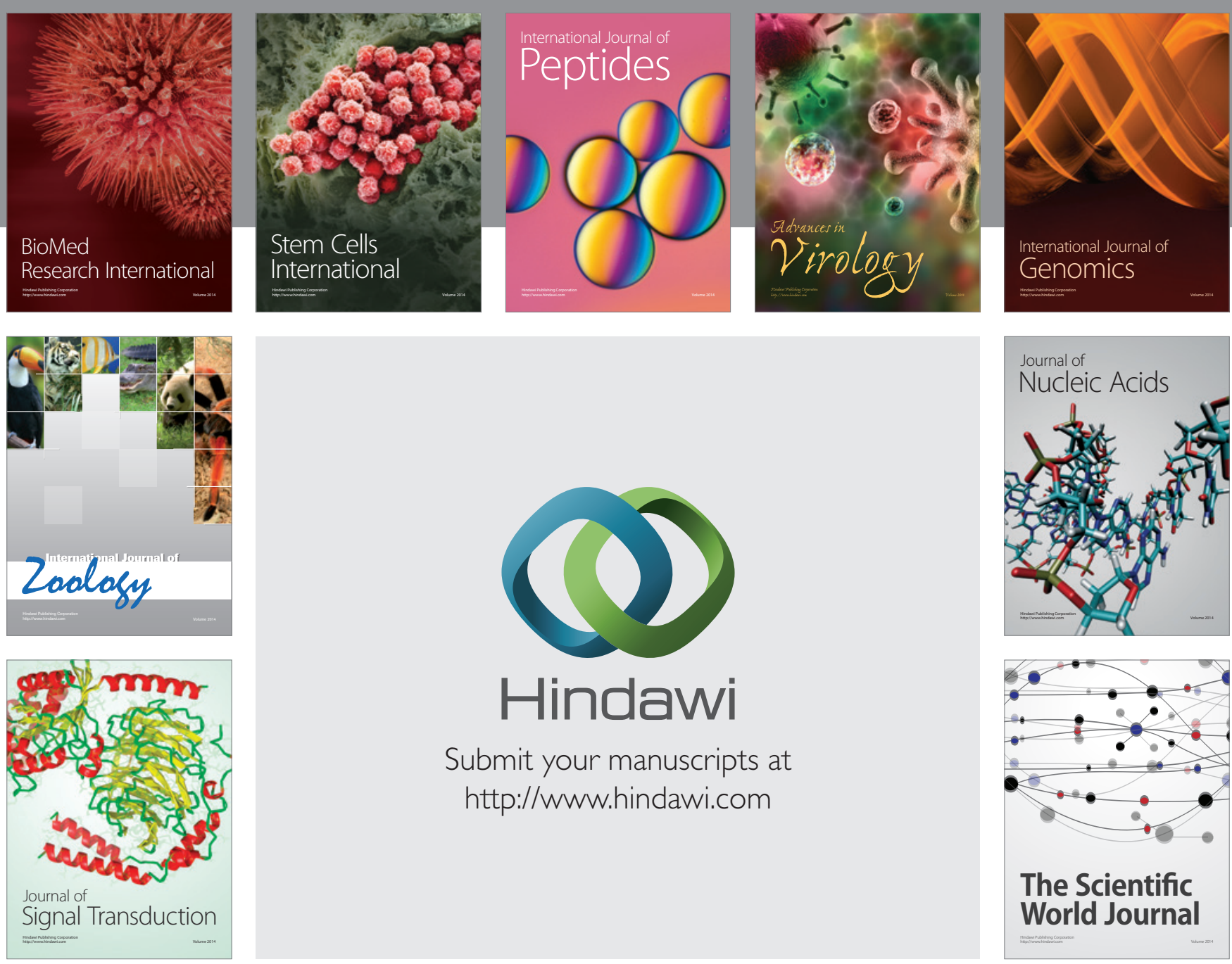

Submit your manuscripts at

http://www.hindawi.com
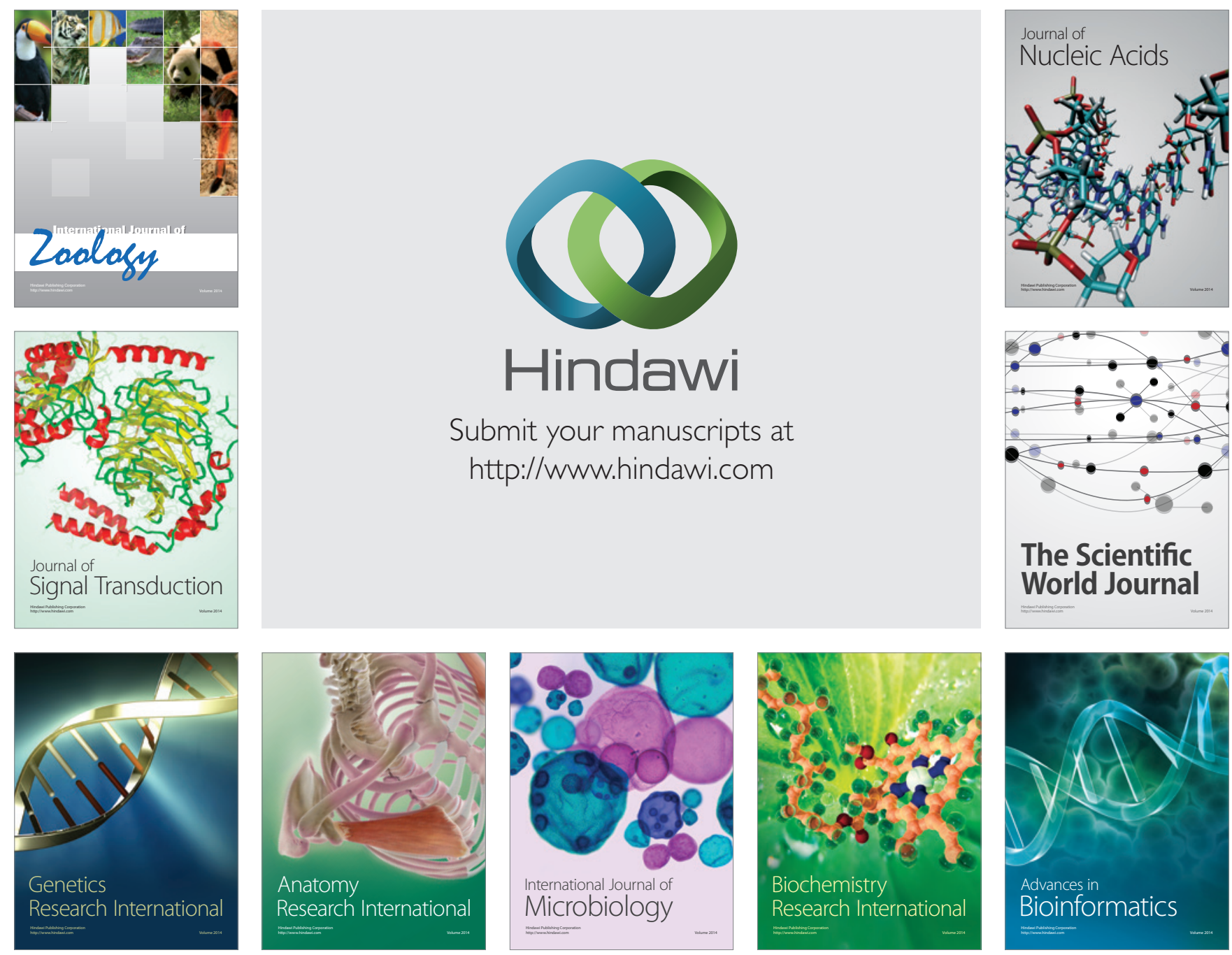

The Scientific World Journal
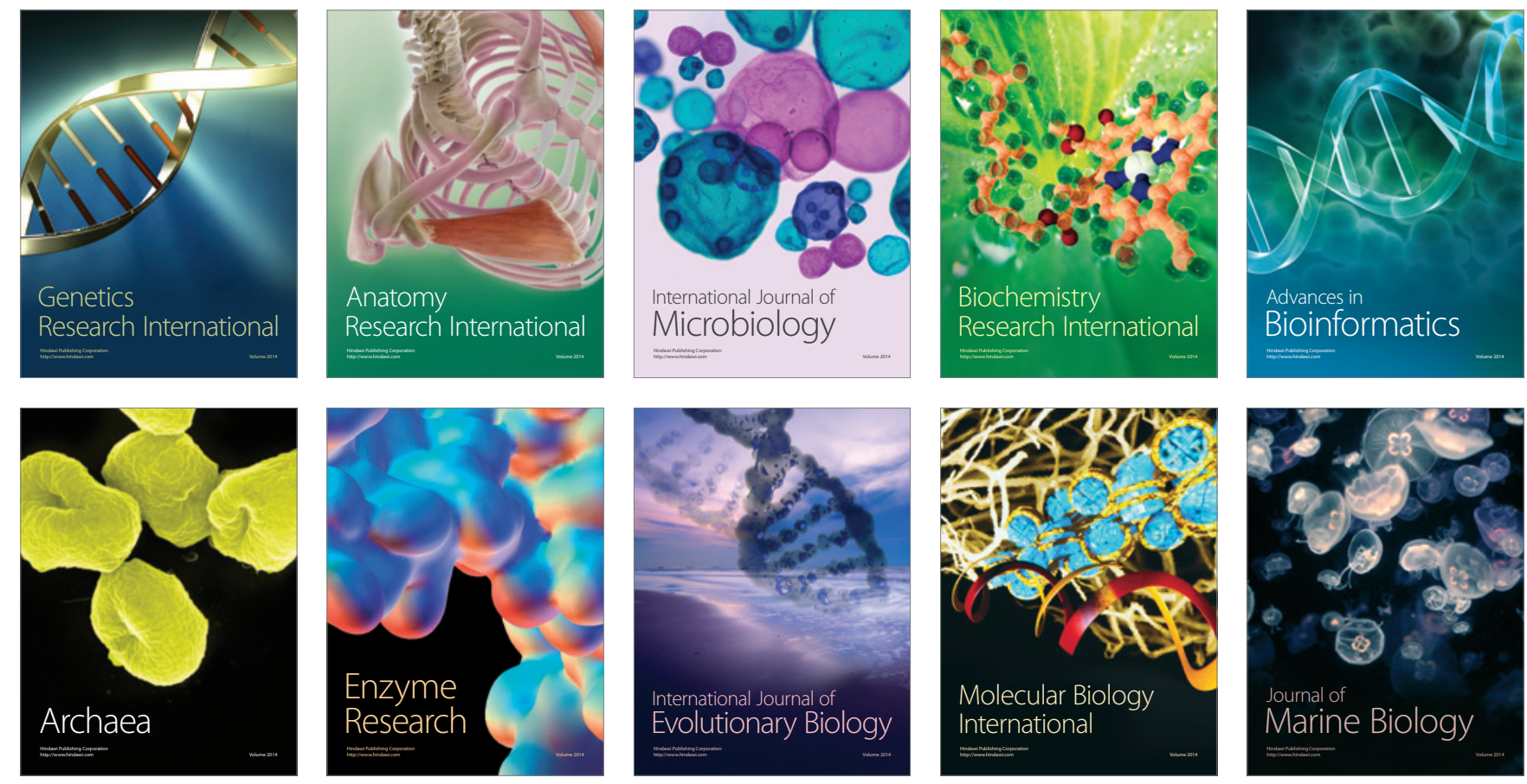